\title{
Risk Factors for Atrial Fibrillation following a Cardiac Surgery
}

\author{
Ibrahim Marai ${ }^{1}$, Wiaam Khatib ${ }^{1}$, Liza Grosman-Rimon ${ }^{2}$, Shemy Carasso ${ }^{3}$, Ali Sakhnini ${ }^{1}$, \\ Edo Birati ${ }^{1}$, and Erez Kachel ${ }^{4}$ \\ ${ }^{1}$ The Baruch Padeh Medical Center Poriya \\ ${ }^{2}$ University Health Network \\ ${ }^{3}$ Poriya Medical Center \\ ${ }^{4}$ B Padeh Medical Center
}

September 25, 2021

\begin{abstract}
Background: Atrial fibrillation (AF) following cardiac surgery is common and has clinical impact on morbidity. The preoperative and intraoperative risk factors are still not well defined. The objective of the study was to examine preoperative and intraoperative risk factors for AF following cardiac surgery. Methods: A retrospective analysis of a database of cardiac surgeries was performed during 2017-2019 at Poriya Medical Center. Preoperative factors and intraoperative were recorded. Results: 208 patients were included in this analysis. Overall AF following cardiac surgery was detected in 50 (24\%) patients. Of 175 patients who did not have history of AF prior to surgery, 27 (15.5\%) had post-operative AF. In the 33 patients with previous AF, AF following surgery was detected in 23 (70\%). Patients with AF following surgery who were older (66.2 \pm 8.0 vs. $60.7 \pm 11.4$ years, $\mathrm{p}=0.002)$, were treated more with anti-arrhythmic drugs $(18.9 \%$ vs $4.5, \mathrm{p}<0.001)$, and had higher rates of pre-operative $\mathrm{AF}(46 \%$ vs $6.3 \%, \mathrm{p}=0.0001)$, prior cerebral vascular accidents $(14 \%$ vs $4.4 \%, \mathrm{p}=0.019)$, and prior valve replacement ( $10 \%$ vs $1.9 \%, \mathrm{p}=0.009$ ) compared to patients without AF following surgery. In multivariate Cox regression analysis, age (HR 1.04, CI 1.01-1.07, $\mathrm{P}=0.006$ ) and history of preoperative AF (HR 6.01, CI 3.42-10.57, $\mathrm{P}<0.001$ ) were predictors of AF following cardiac surgery. The probability of being free of postsurgical AF was $80 \%$ among patients without history of AF compared to $30 \%$ in patients with previous AF history $(\mathrm{p}<0.001)$. Conclusion: Preoperative AF and age were predictors of AF following cardiac surgery
\end{abstract}

\section{Risk Factors for Atrial Fibrillation following a Cardiac Surgery}

Ibrahim Marai ${ }^{1}$, Wiaam Khatib ${ }^{1}$, Liza Grosman-Rimon ${ }^{1}$, Shemy Carasso ${ }^{1}$,Ali Sakhnini ${ }^{1}$, Edo Birati $^{1}$, Erez Kachel $^{2}$

${ }^{1}$ Cardiovascular department, Baruch Padeh Poriya Medical Center, Poriya, The Azrieli Faculty of Medicine, Bar Ilan University, Zefat, Israel

2 Department of cardiac surgery, Baruch Padeh Poriya Medical Center, Poriya, The Azrieli Faculty of Medicine, Bar Ilan University, Zefat, Israel

Running title : Atrial fibrillation post cardiac surgery

Key Words : POAF, Risk Factors, Prediction

Correspondence to :

Ibrahim Marai MD

Cardiovascular department, Baruch Padeh Poriya Medical Center, Poriya, The Azrieli Faculty of Medicine, Bar Ilan University, Zefat, Israel 
imarai@poria.health.gov.il

\begin{abstract}
Background: Atrial fibrillation (AF) following cardiac surgery is common and has clinical impact on morbidity. The preoperative and intraoperative risk factors are still not well defined. The objective of the study was to examine preoperative and intraoperative risk factors for AF following cardiac surgery.

Methods: A retrospective analysis of a database of cardiac surgeries was performed during 2017-2019 at Poriya Medical Center. Preoperative and intraoperative factors were recorded.

Results: 208 patients were included in this analysis. Overall AF following cardiac surgery was detected in $50(24 \%)$ patients. Of 175 patients who did not have history of AF prior to surgery, 27 (15.5\%) had postoperative AF. In the 33 patients with previous AF, AF following surgery was detected in 23 (70\%). Patients with AF following surgery who were older ( $66.2 \pm 8.0$ vs. $60.7 \pm 11.4$ years, $\mathrm{p}=0.002)$, were treated more with anti-arrhythmic drugs $(18.9 \%$ vs $4.5, \mathrm{p}<0.001)$, and had higher rates of pre-operative AF ( $46 \%$ vs $6.3 \%$, $\mathrm{p}=0.0001$ ), prior cerebral vascular accidents ( $14 \%$ vs $4.4 \%, \mathrm{p}=0.019)$, and prior valve replacement ( $10 \%$ vs $1.9 \%, \mathrm{p}=0.009$ ) compared to patients without $\mathrm{AF}$ following surgery. In multivariate Cox regression analysis, age (HR 1.04, CI 1.01-1.07, $\mathrm{P}=0.006$ ) and history of preoperative AF (HR 6.01, CI 3.42-10.57, $\mathrm{P}<0.001$ ) were predictors of AF following cardiac surgery. The probability of being free of postsurgical AF was $80 \%$ among patients without history of AF compared to $30 \%$ in patients with previous AF history $(\mathrm{p}<0.001)$.
\end{abstract}

Conclusion: Preoperative AF and age were predictors of AF following cardiac surgery.

\title{
Background
}

Atrial fibrillation (AF) is a common arrhythmia in the general population and is more common in specific conditions such as cardiac and non-cardiac surgery $[1,2]$. AF following surgery could occur in patients who were or were not previously diagnosed with AF. Post-operative atrial fibrillation (POAF) is defined as the development of $\mathrm{AF}$ in the postoperative period in a patient who was not previously diagnosed with $\mathrm{AF}[2,3]$.

Focusing on POAF following cardiac surgeries is important since it is considered a common complication affecting patients following abundant surgical procedures including coronary artery bypass grafting (CABG), valve surgery, combined CABG and valve surgery, congenital defects surgery, and even percutaneous coronary revascularization but with a much lower risk than major surgical intervention [3-6]. The occurrence of POAF increases in correlation with increasing invasiveness of cardiac surgery, and may reach up to $60 \%$, usually appearing within the first 6 days after surgery with a peak in the $2^{\text {nd }}-4^{\text {th }}$ days [4-6].

POAF is associated with major morbidities such as prolonged mechanical ventilation support, postoperative stroke, myocardial infarction, cardiac arrest, permanent pacemaker, respiratory failure, pneumonia, gastrointestinal complications, acute kidney injury, and a new onset of dialysis. Furthermore, POAF leads to a longer hospital and intensive care unit stay, higher treatment costs, hospital readmission, and most importantly, 2 folds of increased mortality [6-8].

Risk factors that were reported to correlate with increased incidence of POAF, were derived from epidemiological studies, and can be divided into 3 main groups: preoperative, intraoperative and postoperative factors $[9,10]$. In the last 20 years, research has expanded in the field of POAF and several modifications have been implemented to perioperative management and surgical techniques, in an attempt to decrease POAF incidence or its subsequent complications. However, more progress needs to be made [11] . The aim of this study is to evaluate preoperative, intra-operative risk factors and predictors of occurrence of overall AF following cardiac surgery including patients previously diagnosed and who were not previously diagnosed with AF (POAF).

\section{Materials and Methods}

A retrospective (case control) observational study was conducted in the cardiovascular department of Baruch Padeh Medical Center - Poriya to determine predictive risk factors that lead to developing AF in patients 
who had cardiac surgeries. The study was approved by the institutional ethics review board and the research was conducted in accordance with the Helsinki Declaration. Preoperative, intraoperative, and postoperative clinical data and laboratory tests were collected and summarized. Moreover, 30 days after surgery morbidities and mortality were recorded.

The population study included 208 patients who underwent cardiac surgeries at Baruch Padeh Medical Center in the years 2017-2019. Inclusion criteria consisted of: patients undergoing cardiac surgeries in a sinus rhythm with and without a history of AF. Exclusion criteria consisted of: patients with permanent atrial fibrillation, patients who were not in sinus rhythm prior to surgery, and patients with missing data. Patients were divided into 2 groups: patients with and without AF post cardiac surgery irrespective of their AF history prior to cardiac surgery. Pre, intra, and post-operative factors were compared between the 2 groups.

\section{Statistical Methods}

Values were presented as mean and standard deviation, and Categorical variables were represented by percentage and prevalence. Comparison between patients who developed AF post cardiac surgery and those who did not was conducted using t- test or Mann Whitney U for the continues data, and Chi Squared Test for categorical data. Logistic regression analysis was used for identifying pre and intraoperative risk factors for developing AF post cardiac surgery.

Kaplan Meir survival curve was utilized for evaluating the prevalence of AF post-surgery based on the history of pre-surgery AF. P value of less than 0.05 was considered statistically significant.

\section{Results}

208 patients who underwent cardiac surgeries at Baruch Padeh Medical Center in the years 2017-2019 who met the inclusion criteria participated in our study. Overall, AF post cardiac surgery was detected in 50 out of $208(24 \%)$ patients. POAF was detected in $27(15.5 \%)$ out of 175 patients without history of documented AF before cardiac surgery (Table 1). Among the 33 patients with history of AF before cardiac surgery, AF following surgery was detected in $23(70 \%)$ patients.

The participants' demographic and preoperative clinical data are summarized in Table 2. Patients with AF post cardiac surgery were older $(66.2 \pm 8.0$ vs $60.7 \pm 11.4$ years, $\mathrm{p}<0.001)$, utilized more preoperative anti arrhythmic drugs (AADs) (18.9\% vs 4.5\%, p<0.001), and had more AF history (46\% vs 6.3\%, p >0.001), previous cerebrovascular accident (CVA) ( $14 \%$ vs $4.4 \%, \mathrm{p}=0.019)$ and prior valve replacement (10\% vs $1.9 \%$, $\mathrm{p}=0.009$ ) compared to patients without AF post cardiac surgery. Hypertension tended to be more prevalent among patients with AF post cardiac surgery $(74 \%$ vs $59.5 \%, \mathrm{p}=0.06)$. In contrast, gender, height, weight, BMI, BSA, prior beta blockers, diabetes mellitus, dyslipidemia, chronic obstructive pulmonary disease (COPD) and interstitial lung disease, peripheral vascular disease (PVD), renal failure, undergoing dialysis, previous (TIA), coronary vascular disease, congestive heart failure (CHF), ejection fraction (EF), previous $\mathrm{CABG}$, and prior percutaneous coronary intervention (PCI) were not statistically different between the two groups.

Table 3 presents intraoperative factors and laboratory results. Pump time, minimum PH, Pre and postoperative hemoglobin, pre and post-operative creatinine were not statistically different between patients with and without AF following cardiac surgery. Aortic clamp time tended to be longer among patients with AF post cardiac surgery, compared to patients without AF post cardiac surgery (116.7 \pm 52.0 vs $101.7 \pm 50.2$ minutes, respectively, $\mathrm{p}=0.07)$.

Post surgical major bleeding, and infection were not statistically different between the two groups as presented in Table 4. In hospital mortality, including cardiovascular and non-cardiovascular within 30 days and mortality in the first year following the surgery were not statistically different between the two groups. Readmission within 30 days tended to be more prevalent among patients with AF post cardiac surgery compared to patients without AF post cardiac surgery ( $26 \%$ vs $14.6 \%$, respectively , $\mathrm{p}=0.06$ ).

Predictors of AF Post-Cardiac Surgery 
As shown in table 5, univariate Cox regression analysis revealed that age (HR 1.05, CI 1.01-1.08, $\mathrm{P}=0.002$ ) and history of AF (HR 6.48, CI 3.69-11.37, $\mathrm{P}<0.001$ )) were predictors of AF post-surgery. Type of surgery (HR 1.34, CI 0.95-1.91, $\mathrm{p}=0.09$ ) and hypertension (HR 1.72, CI 0.91-3.24, $\mathrm{p}=0.09$ ) tended to predict $\mathrm{AF}$ following cardiac surgery. In multivariate Cox regression analysis, age (HR 1.04, CI 1.01-1.07, P=0.006) and history of preoperative AF (HR 6.01, CI 3.42-10.57, $\mathrm{P}<0.001$ ) were independent predictors of AF following cardiac surgery (table 6).

Kaplan Meir survival curve was utilized for evaluating the probability of AF post-surgery based on the history of AF before surgery (figure 1). The probability of being free of AF post cardiac surgery was $80 \%$ among patients without history of preoperative AF compared to $30 \%$ among patients with history of preoperative $\mathrm{AF}(\mathrm{p}<0.001)$. Most of AF episodes occurred between postoperative 2 and 5 as showed in figure 1.

\section{Discussion}

Overall AF following cardiac surgery was detected in $24 \%$ of patients. POAF (AF following cardiac surgery without history of AF) was detected in $15.5 \%$ of patients without history of AF prior to surgery. Among patients with history of AF, AF following surgery was detected in 70\%. History of AF and age were independent predictors of AF following cardiac surgery.

The true incidence of POAF following cardiac surgery remains unclear due to variability in methods of detection and definitions of POAF [12]. The majority of studies reported POAF rates between 20-40\% following CABG, $40-50 \%$ after valve surgery, up to $60 \%$ following combined valve and CABG surgery, and as high as $80 \%$ following multiple valve surgery [11]. Mariscalco et al. reported that among 17,262 cardiac surgery patients, 4,561 (26.4\%) developed POAF, primarily within 2 days of surgery [13] . Bessissow et al. reported that the incidence of POAF among adults aged $45+$ ranges from $20 \%$ to $40 \%$ among those undergoing thoracic or cardiac surgery [14]. In our study, the overall AF following cardiac surgery was 24\%, and POAF was $15.5 \%$. This rate $(15.5 \%)$ of POAF seems to be lower than the rate reported in the literature if we take into account only patients with no history of AF. The discrepancy could be attributed to differences in definition and detection of POAF. Part of patients undergoing cardiac surgeries who are considered to have no history of AF actually have undetected and or asymptomatic paroxysmal AF before surgery as many of them have risk factors for AF. Thus, to overcome this limitation, we decided to investigate the overall AF following cardiac surgery irrespective of history of AF before surgery.

Many risk factors for POAF were reported in different studies. In general, older patients and those with more preoperative comorbidities are more likely to develop POAF. These risk factors were used to create predictive scoring systems and risk models [2]. Risk models are based on known risk factors such as age, race, CHF, a high EuroSCORE rating, COPD, emergency operation, decreased preoperative left ventricular EF, and decreased estimated glomerular filtration rate (eGFR) [13, 15-17]. However, Current risk prediction models for POAF are derived from epidemiological studies and are not based on pathophysiologic mechanisms. Due to that they are moderately accurate at best, these models are infrequently used in clinical settings [9]. Currently, no validated, evidence-based threshold exists to stratify patients according to risk of developing POAF, and there are no published studies that delineate risk factors for POAF based on significantly differentiating factors.

In general, the major risk factors for POAF among both non-cardiac surgery and cardiac surgery patients are male sex, advancing age, CHF history, and hypertension [14,18]. Among cardiac surgery patients, specifically, history of arrhythmias and AF, history of vascular and coronary artery diseases, valvular heart disease, decreased preoperative kidney function, and type of surgery are also implicated [11,13,14,16,19-23]. Our results are consistent with the previous reports in some aspects. In univariate Cox regression analysis, age and history of AF were found to be predictors of AF post-surgery. Type of surgery and hypertension tended to predict AF following cardiac surgery. In multivariate Cox regression analysis, age and history of preoperative AF were independent predictors of AF following cardiac surgery. Indeed, history of AF was previously reported to be strongly associated with POAF complicating cardiac surgery [24]. However, this report emphasizes the importance of cautious interpretation of the data from different studies as some studies 
included patients with history of arrhythmias and AF. The risk of POAF increases non-linearly with age, and a large retrospective study of nearly 15000 patients who have undergone cardiac surgery showed that POAF incidence increases at higher rates over the age of 55 years [25]. Recently, Eikelboom et al. reported in systemic review and meta-analysis that POAF occurred in 36,988 (23.7\%) out of 155,575 patients [9]. Patients with POAF were older than those without it (mean, 68.7 vs 63.7 years). There were no sex differences between the groups. Hypertension, dyslipidemia, diabetes, and smoking occurred equivalently in patients who did and did not develop POAF [9].With respect to intraoperative factors, there was no difference between patients with and without AF post cardiac surgery, except for aortic clamp time which tended to be longer among patients with AF post cardiac surgery $(\mathrm{p}=0.07)$. In some studies, procedural factors such as the duration of the aortic cross-clamp time, location of the venous cannulation, and duration of cardiopulmonary bypass have been shown to affect the rates of POAF [4].

Finally, POAF after cardiac surgery was reported to be associated with increased short-term and long-term morbidity and mortality, including an eightfold increase in the risk of subsequent AF, as well as a higher rate of stroke and cardiovascular death $[3,7,9,26,27]$. In our study, there was no difference in complications between the two groups, except for readmission within 30 days, which tended to be more frequent among patients with AF following cardiac surgery.

\section{Limitations}

This is a retrospective analysis of a relatively small number of patients. We decided to include all patients with AF following cardiac surgery irrespective of history of AF to overcome the variation in definition of POAF in different studies. Thus, our results applied to all patients with AF following cardiac surgery and not specifically to patients with POAF (patients with no history of AF).

In summary, preoperative $\mathrm{AF}$ and age were independent predictors of AF following cardiac surgery.

\section{References}

1. Lau DH, Linz D, Schotten U, Mahajan R, Sanders P, Kalman JM . Pathophysiology of Paroxysmal and Persistent Atrial Fibrillation: Rotors, Foci and Fibrosis. Heart Lung Circ

. 2017;26:887-893.doi: 10.1016/j.hlc.2017.05.119.

2. Jagadish P, Kirolos I, Khare S, Rawal A, Lin V, Khouzam R. Post-operative atrial fibrillation: should we anticoagulate?. Ann Transl Med, 2019; 7: 407.

3. Dobrev D, Aguilar M, Heijman J, Guichard J, Nattel S. Postoperative Atrial Fibrillation: Mechanisms, manifestations and management. Nature Reviews Cardiology ,2019;16: 417-436.

4. Hashemzadeh H, Dehdilani2 M, Dehdilani1 M. Postoperative Atrial Fibrillation following Open Cardiac Surgery: Predisposing Factors and Complications. Journal of Cardiovascular and Thoracic Research, 2013; 5: 101-107.

5. Greenberg J, Lancaster T, Schuessler R, Melby S. Postoperative atrial fibrillation following cardiac surgery: a persistent complication. European Journal of Cardio-Thoracic Surgery 2017 ;52: 665-672.

6. Zakkar M, Ascione R, James AF, Angelini GD, Suleiman MS. Inflammation, oxidative stress and postoperative atrial fibrillation in cardiac surgery. Pharmacology \& Therapeutics 2015; 154:13-20.

7. Megens M, Churilov L, Thijs V. New-Onset Atrial Fibrillation after Coronary Artery Bypass Graft and Long-Term Risk of Stroke: A Meta-Analysis. Journal of the American Heart Association, 2019; 6: e007558.

8. Lapar D, Speir A, Crosby I, Fonner E, Brown M, Rich J, et al. Postoperative Atrial Fibrillation Significantly Increases Mortality, Hospital Readmission, and Hospital Costs. The Annals of Thoracic Surgery, 2014; 98 $: 527-533$.

9. Eikelboom R, Sanjanwala R., Le M., Yamashita M., Arora R. Postoperative Atrial Fibrillation After Cardiac Surgery: A Systematic Review and Meta-Analysis. Ann Thorac Surg, 2021;111: 544-554. 
10. Jakobsen $\varnothing$, Næsheim T, Nerg K, Dag S, Steensrud T. Adenosine instead of supranormal potassium in cardioplegia: It is safe, efficient, and reduces the incidence of postoperative atrial fibrillation. A randomized clinical trial. The Journal of Thoracic and Cardiovascular Surgery, $2013 ; 145$ :812-818.

11. Gudbjartsson T, Helgadottir S, Sigurdsson M, Taha A, Jeppsson A, Christensen T, et al. New-onset postoperative atrial fibrillation after heart surgery. Acta anaesthesiologica Scandinavica, 2020; 64 :145-155.

12. Maisel WH, Rawn JD, Stevenson WG. Atrial fibrillation after cardiac surgery. Ann Intern Med. 2001; 135:1061-1073.

13. Mariscalco G, Biancari F, Zanobini M, Cottini M, Piffaretti G, Saccocci M, et al. Bedside tool for predicting the risk of postoperative atrial fibrillation after cardiac surgery: the POAF score. J Am Heart Assoc, 2014 ; 3: e000752.

14. Bessissow A, Khan J, Devereaux PJ, Alvarez-Garcia J, Alonso-Coello P, et al. Postoperative atrial fibrillation in non-cardiac and cardiac surgery: an overview. J Thromb Haemost, 2015;13 Suppl 1:S304-312.

15. Helgadottir S, Sigurdsson MI, Ingvarsdottir IL, Arnar DO, Gudbjartsson T. Atrial fibrillation following cardiac surgery: risk analysis and long-term survival. J Cardiothorac Surg. 2012; 7:87.

16. Zaman AG, Archbold RA, Helft G, Paul EA, Curzen NP, Mills PG. Atrial fibrillation after coronary artery bypass surgery: a model for preoperative risk stratification. Circulation. 2000; 101:1403-1408.

17. Waldron NH, Cooter M, Piccini JP, Anstrom K, Klinger R, Kertai M, et al. Predictive ability of perioperative atrial fibrillation risk indices in cardiac surgery patients: a retrospective cohort study. Can J Anaesth. 2018; 65:786-796.

18. Bhave PD, Goldman LE, Vittinghoff E, et al. Incidence, predictors, and outcomes associated with postoperative atrial fibrillation after major noncardiac surgery. Am Heart J. 2012; 164:918-924.

19. Aranki SF, Shaw DP, Adams DH, et al. Predictors of atrial fibrillation after coronary artery surgery. Current trends and impact on hospital resources. Circulation. 1996; 94:390-397.

20. Steinberg JS. Postoperative atrial fibrillation: a billion-dollar problem. J Am Coll Cardiol. 2004; 43:10011003.

21. Ak K, Akgun S, Tecimer T, et al. Determination of histopathologic risk factors for postoperative atrial fibrillation in cardiac surgery. Ann Thorac Surg. 2005; 79:1970-1975.

22. Zacharias A, Schwann TA, Riordan CJ, Durham SJ, Shah AS, Habib RH. Obesity and risk of new-onset atrial fibrillation after cardiac surgery. Circulation. 2005; 112:3247-3255.

23. Muehlschlegel JD, Burrage PS, Ngai JY, et al. Society of Cardiovascular Anesthesiologists/European Association of Cardiothoracic Anaesthetists practice advisory for the Management of Perioperative Atrial Fibrillation In Patients Undergoing Cardiac Surgery. Anesth Analg. 2019;128:33-42.

24. Funk, M., Richards, S. B., Desjardins, J., Bebon, C. \& Wilcox, H. Incidence, timing, symptoms, and risk factors for atrial fibrillation after cardiac surgery. Am. J. Crit. Care 12, 2003; 12:424-433.

25. Shen J, Lall S, Zheng V, Buckley P, Damiano RJ Jr, Schuessler RB. The persistent problem of new-onset postoperative atrial fibrillation: a single-institution experience over two decades. J Thorac Cardiovasc Surg. 2011; 141:559-570.

26. Phan K, Ha HS, Phan S, Medi C, Thomas SP, Yan TD. New-onset atrial fibrillation following coronary bypass surgery predicts long-term mortality: a systematic review and meta-analysis. Eur J Cardiothorac Surg. 2015; 48:817-824.

27. Kosmidou I, Stone GW. New-onset atrial fibrillation after PCI and CABG for left main disease: insights from the EXCEL trial and additional studies. Curr Opin Cardiol. 2018; 33:660-664. 
Table 1: Atrial fibrillation post-cardiac surgery

\begin{tabular}{llll}
\hline & $\begin{array}{l}\text { No atrial fibrillation post } \\
\text { surgery }(\mathrm{n}=158)\end{array}$ & $\begin{array}{l}\text { Atrial fibrillation post } \\
\text { surgery }(\mathrm{n}=50)^{*}\end{array}$ & P value \\
\hline $\begin{array}{l}\text { History of atrial } \\
\text { fibrillation }(\mathrm{n}=33)\end{array}$ & $10(6.3 \%)$ & $23(46 \%)$ & $<0.001$ \\
$\begin{array}{l}\text { No history of atrial } \\
\text { fibrillation }(\mathrm{n}=175)\end{array}$ & $148(93.7 \%)$ & $27(54 \%) * *$ & \\
\hline
\end{tabular}

${ }^{*}$ Atrial fibrillation post cardiac surgery $=50$ out of $208(24 \%) . * * \mathrm{POAF}=27$ out of $175(15.5 \%)$

Table 2: Demographic and preoperative clinical data

\begin{tabular}{|c|c|c|}
\hline & No atrial fibrillation post surgery $(n=158)$ & Atrial fibrillation post surg \\
\hline Male $\mathrm{n}(\%)$ & $125(79.1 \%)$ & $33(70 \%)$ \\
\hline Female $\mathrm{n}(\%)$ & $33(20.9 \%)$ & $15(30 \%)$ \\
\hline Age (years) & $60 \pm 11$ & $66 \pm 8$ \\
\hline Height $(\mathrm{cm})$ & $169 \pm 8$ & $169 \pm 10$ \\
\hline Weight $(\mathrm{Kg})$ & $80.4 \pm 14.6$ & $81.1 \pm 12.7$ \\
\hline $\operatorname{BMI}\left(\mathrm{kg} / \mathrm{m}^{2}\right)$ & $27.8 \pm 4.3$ & $28.3 \pm 3.5$ \\
\hline $\mathrm{BSA}\left(\mathrm{m}^{2}\right)$ & $1.9 \pm 0.1$ & $1.9 \pm 0.1$ \\
\hline Beta blockers n(\%) & $90(7.3 \%)$ & $33(67.3 \%)$ \\
\hline Pre-operative antiarrhythmic drugs n(\%) & $7(4.5 \%)$ & $9(18.4 \%)$ \\
\hline Hypertension $\mathrm{n}(\%)$ & $94(59.5 \%)$ & $37(74 \%)$ \\
\hline Diabetes Mellitus treated with insulin n(\%) & $31(19.6 \%)$ & $6(12 \%)$ \\
\hline Diabetes Mellitus not treated with insulin $\mathrm{n}(\%)$ & $39(24.7 \%)$ & $11(22 \%)$ \\
\hline Dyslipidemia $\mathrm{n}(\%)$ & $105(66.5 \%)$ & $32(64 \%)$ \\
\hline COPD and Interstitial lung disease n(\%) & $7(4.4 \%)$ & $1(2 \%)$ \\
\hline Peripheral Vascular Disease $\mathrm{n}(\%)$ & $5(3.2 \%)$ & $1(2 \%)$ \\
\hline Renal failure $\mathrm{n}(\%)$ & $5(3.2 \%)$ & $3(6.1 \%)$ \\
\hline Patients undergoing dialysis $\mathrm{n}(\%)$ & $2(1.3 \%)$ & $0(0 \%)$ \\
\hline Permanent pacemaker $\mathrm{n}(\%)$ & $3(1.9 \%)$ & $1(2 \%)$ \\
\hline Previous CVA $n(\%)$ & $7(4.4 \%)$ & $7(14 \%)$ \\
\hline Previous TIA $\mathrm{n}(\%)$ & $1(0.6 \%)$ & $1(2 \%)$ \\
\hline Coronary Vascular disease $\mathrm{n}(\%)$ & $97(61.4 \%)$ & $32(64 \%)$ \\
\hline Chronic heart failure $\mathrm{n}(\%)$ & $8(5.1 \%)$ & $4(8 \%)$ \\
\hline Ejection Fraction $(\%)$ & $53.6 \pm 16.3$ & $52.5 \pm 23.6$ \\
\hline Previous CABG $\mathrm{n}(\%)$ & $3(1.9 \%)$ & $2(4 \%)$ \\
\hline Prior Valve Replacement $\mathrm{n}(\%)$ & $3(1.9 \%)$ & $5(10 \%)$ \\
\hline Prior PCI n(\%) & $43(27.2 \%)$ & $16(32 \%)$ \\
\hline
\end{tabular}

Table 3: Intra-operative factors and laboratory results:

\begin{tabular}{lll}
\hline & No atrial fibrillation post surgery $(\mathrm{n}=158)$ & Atrial fibrillation post surgery $(\mathrm{n}=$ \\
\hline Pump Time $(\min )$ & $130.6 \pm 59.2$ & $144.9 \pm 17.6$ \\
Aortic clamp time $(\min )$ & $101.7 \pm 50.2$ & $116.7 \pm 52.0$ \\
Minimum PH & $7.2 \pm 0$ & $7.2 \pm 0$ \\
Pre-operative Hemoglobin $(\mathrm{g} / \mathrm{L})$ & $13.3 \pm 1.9$ & $13.3 \pm 1.8$ \\
Pre-operative Creatinine $(\mathrm{mg} / \mathrm{dL})$ & $0.9 \pm 0.6$ & $0.9 \pm .25$
\end{tabular}




\begin{tabular}{lll}
\hline & No atrial fibrillation post surgery $(\mathrm{n}=158)$ & Atrial fibrillation post surgery $(\mathrm{n}=$ \\
\hline Post-operative Hemoglobin $(\mathrm{g} / \mathrm{L})$ & $10.8 \pm 1.9$ & $10.9 \pm 1.3$ \\
Post-operative Creatinine $(\mathrm{mg} / \mathrm{dL})$ & $0.9 \pm 0.8$ & $0.9 \pm 0.2$ \\
Post-operative Hemoglobin, Day1 $(\mathrm{g} / \mathrm{L})$ & $9.9 \pm 1.2$ & $9.5 \pm 1.7$ \\
Post-operative Creatinine, Day1 $(\mathrm{mg} / \mathrm{dL})$ & $0.9 \pm 0.4$ & $0.9 \pm 0.3$ \\
\hline
\end{tabular}

Table 4: Post-operative complications

\begin{tabular}{lll}
\hline & No atrial fibrillation post surgery $(\mathrm{n}=158)$ & Atrial fibrillation post surger \\
\hline Major Bleeding* $\mathrm{n}(\%)$ & $7(4.4 \%)$ & $3(6 \%)$ \\
Infection $\mathrm{n}(\%)$ & $2(1.3 \%)$ & $2(4 \%)$ \\
Cardio-vascular disease (CVD) mortality $\mathrm{n}(\%)$ & $6(3.8 \%)$ & $1(2 \%)$ \\
Non -CVD mortality $\mathrm{n}(\%)$ & $1(0.6 \%)$ & $0(0 \%)$ \\
Mortality within 30 days $\mathrm{n}(\%)$ & $7(4.4 \%)$ & $1(2 \%)$ \\
Mortality in 1 year n(\%) & $9(5.7 \%)$ & $3(6 \%)$ \\
Readmission within 30 days $\mathrm{n}(\%)$ & $23(14.6 \%)$ & $9(26 \%)$ \\
Multiple readmissions $\mathrm{n}(\%)$ & $31(19.6 \%)$ & $9(18 \%)$ \\
\hline
\end{tabular}

*blood transfusion is needed

Table 5: Univariable Cox regression of predictors of AF post-surgery.

\begin{tabular}{llll}
\hline & B Coefficients & Hazard Ratio $(\mathrm{CI})$ & P Value \\
\hline Age & 0.05 & $1.05(1.01-1.08)$ & 0.002 \\
Gender & 0.41 & $1.51(0.82-2.76)$ & 0.18 \\
Beta blockers & 0.38 & $1.47(0.81-2.67)$ & 0.20 \\
Surgery Type & 0.29 & $1.34(0.95-1.91)$ & 0.09 \\
History of atrial fibrillation & 1.86 & $6.48(3.69-11.37)$ & $<0.001$ \\
Pump time & 0.00 & $1.00(0.99-1.00)$ & 0.18 \\
Aortic clamp time & 0.00 & $1.00(0.99-1.00)$ & 0.11 \\
Minimum PH & 2.64 & $14.05(0.10-1870.71)$ & 0.29 \\
BMI & 0.014 & $1.01(0.95-1.08)$ & 0.66 \\
Hypertension & 0.54 & $1.72(0.91-3.24)$ & 0.09 \\
Chronic heart failure & 0.39 & $1.48(0.53-4.13)$ & 0.44 \\
\hline
\end{tabular}

Table 6: Multivariable Cox regression predictors of AF post-surgery.

\begin{tabular}{llll}
\hline & B Coefficients & Hazard Ratio (CI) & P Value \\
\hline Age & 0.04 & $1.04(1.01-1.07)$ & 0.006 \\
History of atrial fibrillation & 1.79 & $6.01(3.42-10.57)$ & $<0.001$ \\
\hline
\end{tabular}




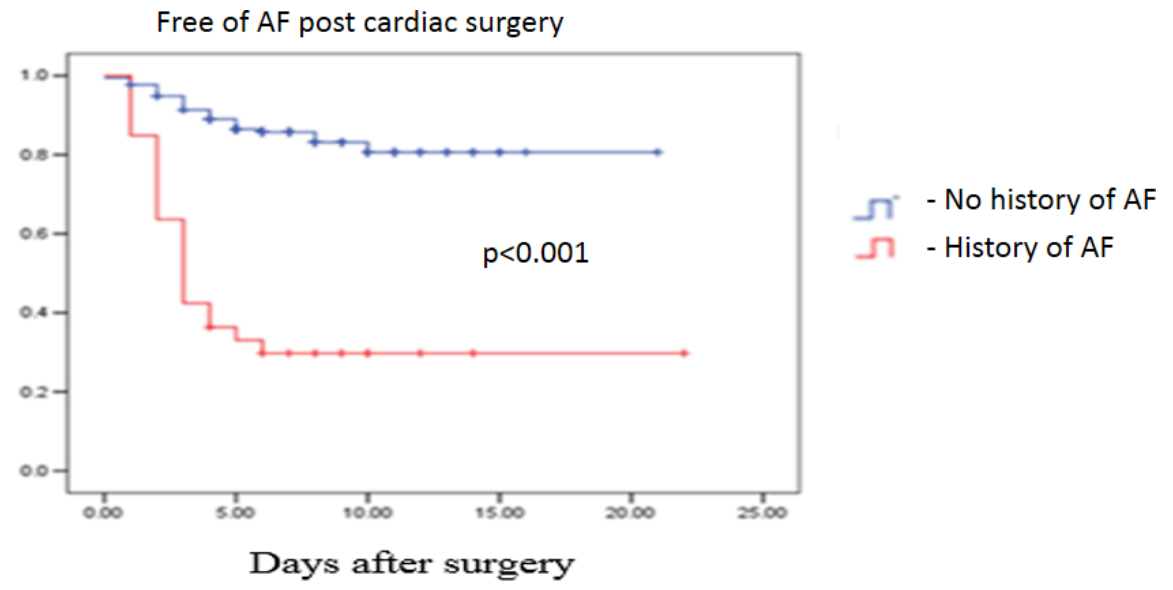

Figure 1: Kaplan Meir survival curve evaluating the probability of AF post-surgery based on the history of AF before surgery.

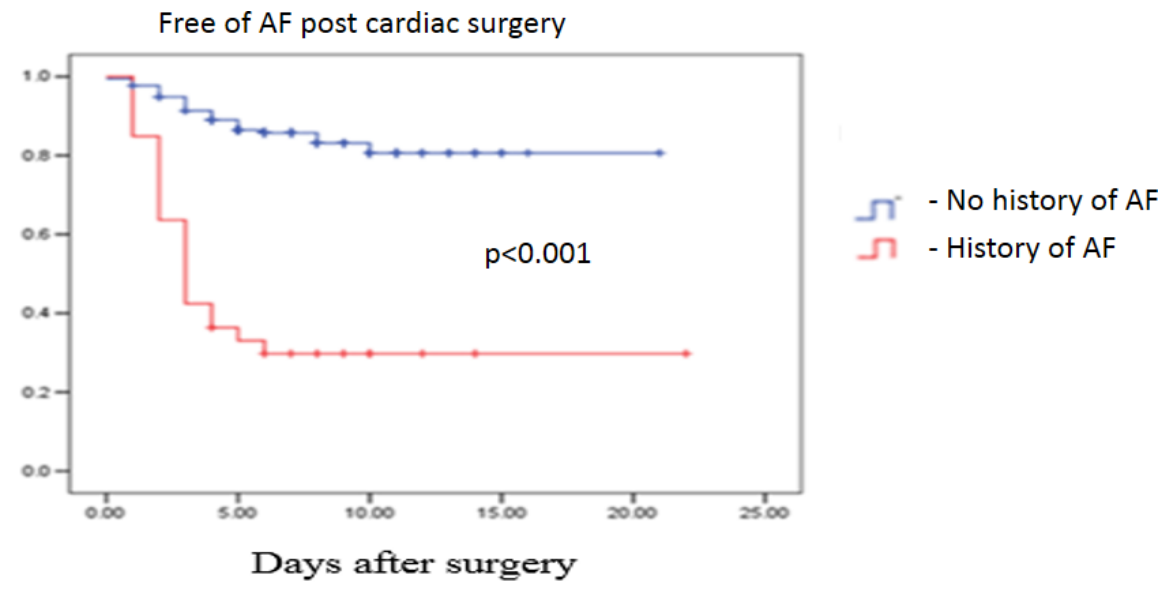

